\title{
Plasma from human mothers of fetuses with severe arthrogryposis multiplex congenita causes deformities in mice
}

\author{
Leslie Jacobson, ${ }^{1}$ Agata Polizzi, ${ }^{1}$ Gillian Morriss-Kay, ${ }^{2}$ and Angela Vincent ${ }^{1}$ \\ ${ }^{1}$ Neurosciences Group, Institute of Molecular Medicine, John Radcliffe Hospital, Oxford OX3 9DS, United Kingdom \\ ${ }^{2}$ Department of Human Anatomy and Genetics, University of Oxford, Oxford, United Kingdom.
}

Address correspondence to: Angela Vincent, Institute of Molecular Medicine, John Radcliffe Hospital, Oxford OX3 9DS, United Kingdom. Phone: 44-1865-222322; Fax: 44-1865-222402; E-mail: angela.vincent@imm.ox.ac.uk

Leslie Jacobson's present address is: Department of Biochemistry, Hellenic Pasteur Institute, 115.21 Athens, Greece. Agata Polizzi's present address is: Department of Pediatric Neurology, University of Catania, I-95125 Catania, Italy.

Agata Polizzi and Leslie Jacobson contributed equally to this work.

Received for publication December 1, 1998, and accepted in revised form February 23, 1999.

\begin{abstract}
Arthrogryposis multiplex congenita (AMC) is characterized by fixed joint contractures and other deformities, sometimes resulting in fetal death. The cause is unknown in most cases, but some women with fetuses affected by severe AMC have serum antibodies that inhibit fetal acetylcholine receptor (AChR) function, and antibodies to fetal antigens might play a pathogenic role in other congenital disorders. To investigate this possibility, we have established a model by injecting pregnant mice with plasma from four anti-AChR antibody-positive women whose fetuses had severe AMC. We found that human antibodies can be transferred efficiently to the mouse fetus during the last few days of fetal life. Many of the fetuses of dams injected with AMC maternal plasmas or Ig were stillborn and showed fixed joints and other deformities. Moreover, similar changes were found in mice after injection of a serum from one anti-AChR antibody-negative mother who had had four AMC fetuses. Thus, we have confirmed the role of maternal antibodies in cases of AMC associated with maternal anti-AChR, and we have demonstrated the existence of pathogenic maternal factors in one other case. Importantly, this approach can be used to look at the effects of other maternal human antibodies on development of the fetus.
\end{abstract}

J. Clin. Invest.103:1031-1038 (1999).

\section{Introduction}

Arthrogryposis multiplex congenita (AMC) is a well-recognized congenital disorder characterized by contractures in more than one joint, with or without other abnormalities, and is sometimes associated with severe fetal maldevelopment and fetal or neonatal death. Although the incidence is usually given as 1 in 3,000 births, some forms of joint contractures (e.g., bilateral talipes) or skeletal deformities such as scoliosis are much more common. AMC can be caused by infectious agents, drugs, toxins, or physical agents, as well as genetic factors and maternal disease $(1,2)$. The pathology is variable (2-4), but the common final pathway appears to be lack or restriction of fetal movement in utero (5-7).

We previously reported high levels of antibodies against human muscle acetylcholine receptor (AChR) in five women with histories of AMC recurring in successive pregnancies $(8,9)$. The fetuses, which were mostly stillborn or terminated for fetal anomalies, showed dysmorphic facies and lung hypoplasia as well as joint contractures, often referred to as the Pena-Shokeir syndrome (10). Anti-AChR antibodies are usually associated with acquired myasthenia gravis (MG), a condition in which weakness and fatigue result from loss of AChRs from the neuromuscular junction (reviewed in ref. 11). Transient neonatal MG sometimes occurs in neonates born to mothers with MG, due to placental transfer of the anti- bodies (11-13). However, three of the mothers (AMC-Ms) of babies with AMC were asymptomatic or had mild or unrecognized MG at the time that their babies were affected $(8,9,14)$, suggesting that the anti-AChR antibodies were different from those usually associated with MG. Indeed, the serum and IgG from these women blocked, by $>90 \%$, the function of fetal AChR expressed in the human muscle-like cell line, TE671, and in Xenopus oocytes $(8,9)$. They did not, however, block the function of adult AChR, explaining the marked effects on the fetuses and the relative sparing of their mothers (in humans, fetal AChR is replaced by the adult form by 33 weeks' gestation; ref. 15).

These observations led us to propose $(8,9)$, as others have done previously $(16,17)$, that other fetal abnormalities might be caused by maternal antibodies to fetal antigens or to neuronal antigens that are exposed during development. To test this hypothesis, we have established a mouse model of maternal-fetal transfer of human antibodies and demonstrated that transfer of antibodies from AMC-Ms results in the appearance of AMC features in the mouse pups. Some of this work has appeared previously in abstract form $(18,19)$.

\section{Methods}

Clinical material. Plasmas were obtained after plasma exchange from four women with histories of severe AMC in their babies and from one nongravid woman with typical MG. Clinical 
Table 1

Clinical and serological features of mothers with AMC

\begin{tabular}{|c|c|c|c|c|c|c|}
\hline Mother & No. pregnancies & MG, when diagnosed & $\begin{array}{c}\text { No. of fetuses/neonates } \\
\text { with typical features } \\
\text { of } A M C^{A}\end{array}$ & $\begin{array}{l}\text { Additional features noted at } \\
\text { postmortem examination }\end{array}$ & $\begin{array}{l}\text { Antibody to } \\
\text { human fetal } \\
\text { AChR }(n M)\end{array}$ & $\begin{array}{l}\text { Antibody tc } \\
\text { human adu } \\
\text { AChR (nM }\end{array}$ \\
\hline AMC-M1 & 7 & No & $\begin{array}{l}6 \text { (includes } 5 \text { terminated } \\
\text { for lack of fetal } \\
\text { movement) }\end{array}$ & $\begin{array}{l}\text { Scoliosis, shrinkage of the } \\
\text { right side of the brain and } \\
\text { necrosis of the cerebellum noted } \\
\text { in one fetus at postmortem }\end{array}$ & 210 & 90 \\
\hline AMC-M2 & 5 & Yes, after 4th baby & 4 & $\begin{array}{l}\text { Cryptorchidism, absent } \\
\text { stomach, CNS atrophy }\end{array}$ & 330 & 150 \\
\hline AMC-M6 & 2 & Yes, after 2nd baby & $\begin{array}{l}2 \text { (includes } 1 \\
\text { terminated for lack } \\
\text { of fetal movement) }\end{array}$ & $\begin{array}{c}\text { Hydrops, scoliosis, pectus } \\
\text { excavatum, abnormal genitalia, } \\
\text { ascites, myositis, CNS anomalies }\end{array}$ & 360 & 40 \\
\hline AMC-M7 & 2 & Yes, before 1st baby & 2 & $\begin{array}{c}\text { Hypotonia, esophageal atresia, } \\
\text { cleft lip/palate }\end{array}$ & 3000 & 1350 \\
\hline
\end{tabular}

Date from ref. 14; Brueton et al., manuscript in preparation; and Newsom-Davis, unpublished observations. Cases described as AMC-M3, AMC-M4, and AMC-M5 in ref. 11 were not studied because of insufficient serum samples. Ancludes fixed joints, dysmorphic facies, lung hypoplasia, hydramnios, and hydrops. AMC, arthrogryposis multiplex congenita; CNS, central nervous system; $M G$, myasthenia gravis.

details of the AMC-Ms are given in Table 1. In addition, serum from a woman with a history of four fetuses with fatal AMC was sent for testing by E. Whittaker (Washington, DC, USA). Control plasmas were donated by healthy laboratory workers (HC), including three women who had had at least two pregnancies within the preceding 10 years (Multip). Other neurologic control samples (OND) were obtained from therapeutic plasma exchange. Ethical approval for use of these materials was given by the Central Oxford Research Ethics Committee. Purified IgG was obtained from two plasmas by affinity chromatography using protein G-Sepharose (Pharmacia Ltd., Uppsala, Sweden), and a crude Ig fraction was concentrated from plasma using precipitation with $40 \%$ saturate ammonium sulfate. They were extensively dialyzed against Hartman's solution before injection.

Anti-AChR antibody measurements in serum and fetal extracts. Muscle or TE671 cell line extracts were labeled with ${ }^{125} \mathrm{I}$ - $\alpha$-bungarotoxin ( ${ }^{125} \mathrm{I}-\alpha$-BuTx$)$ and used as described previously (20). Fetal extracts were made by homogenizing unfixed whole fetuses in $50 \mathrm{mM}$ TRIS buffer with $150 \mathrm{mM} \mathrm{NaCl}, 1 \mathrm{mM}$ EDTA, 1 mM EGTA and $0.2 \mathrm{mM}$ PMSF. The homogenate was spun to remove insoluble material, and the supernatant was stored at $-20^{\circ} \mathrm{C}$ until required. To measure anti-AChR antibody, dilutions of purified IgG, crude Ig fractions, plasma, mouse sera, or undiluted fetal extracts (usually $25 \mu \mathrm{l}$ ) were incubated overnight with $50-\mu \mathrm{l}$ aliquots of ${ }^{125} \mathrm{I}-\alpha$-BuTx-labeled muscle preparations containing approximately $30 \mathrm{fmol}$ of AChR. Normal human serum $(1 \mu \mathrm{l})$ was added to provide carrier IgG, and immunoprecipitation was performed with goat anti-human Ig. The cpm in the pellet were expressed as nanomoles of ${ }^{125} \mathrm{I}-\alpha$-BuTx binding sites precipitated, after subtraction of $\mathrm{cpm}$ precipitated in the presence of carrier serum alone.

Animal breeding and injection. MF1 female mice (5-8 weeks old) and adult stud males were obtained from Harlan UK Ltd.(Oxfordshire, United Kingdom) and housed under standard laboratory conditions before and after mating. E0 was defined as the day on which a vaginal plug was detected. After successful mating, the pregnant mice were kept in pairs until E9; thereafter, they were caged separately.

Injections of adult mice with human plasma or Ig preparations have become a routine procedure for investigating the effects of autoantibodies on adult muscle neuromuscular func- tion (21-23). Pregnant mice were injected with $0.5 \mathrm{ml}$ of plasma (or equivalent amounts of IgG or Ig) intraperitoneally from E9 to E13 and subcutaneously from E14 to E16. Injections were never performed for more than 8 consecutive days, and the dams showed no apparent ill effects. After spontaneous delivery, the pups were left with the mother and their progress was monitored for up to 6 weeks. In many cases, the mothers were sacrificed at E18/19 by exsanguination under anesthesia followed by dislocation of the neck. The abdomen was opened to expose the uterus. The number of fetuses and intrauterine deaths in each horn of the uterus was recorded. The extent of fetal movements, both spontaneous and after mechanical stimulation, was noted. The uterus was then removed and the fetuses dissected out, separated from the placentas, washed in warm water, observed for spontaneous and mechanically stimulated movements including respiratory efforts, examined for signs of deformities, and photographed. The number and combined weight of the fetuses were recorded.

Fetuses were sacrificed by fixation in Bouin's fluid. After $18-24 \mathrm{~h}$, they were rinsed and stored in $70 \%$ ethanol or were placed directly in $95 \%$ ethanol. They were photographed, either alive or after fixation, by the Department of Histopathology at John Radcliffe Hospital. For histology, the Bouin's-fixed fetuses were embedded in paraffin, sectioned at $10 \mu \mathrm{m}$, and stained with hematoxylin and eosin.

\section{Results}

Clinical details of mothers of babies with AMC. Each of the AMC-Ms had had at least two consecutive affected fetuses (see Table 1). Most of the babies had fixed joints, lung hypoplasia, and facial abnormalities, but additional features were also present, such as cutaneous edema/hydrops, pectus excavatum, scoliosis, abnormal genitalia, ascites, myositis, and central nervous system (CNS) atrophy; these features are not uncommon in association with AMC (1-4). In addition, AMC-M7's first baby had esophageal atresia and cleft lip and palate. Interestingly, both AMC-M1 and AMC-M2 have surviving children with mild sensorineural deafness (ref. 14; and Brueton, L., et al., manuscript in preparation). 

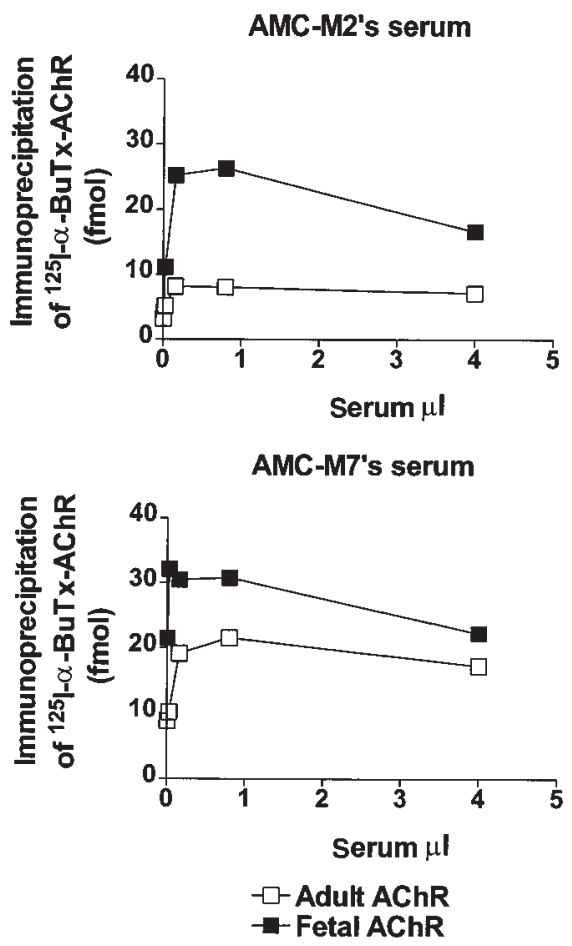

Figure 1

Anti-AChR antibodies tested for binding to adult and fetal human acetylcholine receptor $(A C h R)$ in plasma from AMC-M2 and AMC-M7. Approximately equal amounts of fetal AChR (from TE671 cells) and adult AChR (from TE671- $\varepsilon$, a subline that expresses predominantly the adult form; ref. 20) were used. Dilutions of each plasma were added to the ${ }^{125} \mathrm{I}-\alpha$ bungarotoxin ( ${ }^{125} /$ - $\left.\alpha B u T x\right)$-labeled AChR preparations for $2 \mathrm{~h}$, followed by immunoprecipitation with goat anti-human IgG. Results are given as femtomoles of ${ }^{125}$ I- $\alpha$ bungarotoxin AChR precipitated by each dilution of serum. All antibody measurements in this study are based on titrations similar to these. AMC, arthrogryposis multiplex congenita.

Importantly, AMC-M1 has never had symptoms of MG and has been healthy throughout. Plasma exchange during her sixth affected pregnancy prolonged fetal movements but did not prevent development of AMC (8). AMC-M2 and AMC-M6 were not diagnosed as having MG until after their fourth (14) and second affected babies, respectively, but subsequently, after thymectomy for MG and immunosuppressive treatment for their MG symptoms, each has given birth to a healthy baby (Huson, S., and NewsomDavis, J., unpublished observations).

Reactivity of AMC-M sera with adult and fetal AChR. Plasmas were obtained from the first bag of plasma exchange, and before any other immunosuppressive treatment, and were first tested for antibodies to human adult and fetal AChR. Figure 1 shows examples of immunoprecipitation of AChR, labeled with ${ }^{125} \mathrm{I}$ $\alpha \operatorname{BuTx}$ (a snake toxin that binds strongly to the AChR), by AMC-M2 and AMC-M7 plasmas. AMC-M7 displayed the highest titer of antibody against human AChR, and all four plasmas reacted more strongly with fetal AChR than with adult AChR (Table 1). In addition, all four plasmas at 1:100 dilution inhibited fetal AChR function by $>90 \%$ as described previously for AMC-M1 and AMC-M2 $(8,9)$.
Establishing transfer of buman IgG. To test whether human IgG antibodies were transferred to the mouse fetus, we first injected a series of pregnant dams with antiAChR-positive plasma from a female, nongravid patient with typical MG at $0.5 \mathrm{ml} /$ day from gestational day $\mathrm{E} 10$ to E17. None of the dams showed any signs of weakness. At intervals, individual dams were exsanguinated under anesthesia; the fetuses were removed, and an extract of fetal body fluid was tested for anti-AChR antibodies. The amount of fetal body extract used $(25 \mu \mathrm{l})$ was shown in separate experiments to be equivalent to about $3.5 \mu \mathrm{l}$ of fetal serum (data not shown). The transfer of antibody to the fetuses was efficient as illustrated in Figure $2 a$. The fetal anti-AChR began to rise soon after the injections were started and reached appreciable levels by E18; this was mirrored by a fall in anti-AChR in the dams' sera during the injection period (Figure $2 a$ ), probably partly reflecting the large increase in the maternal/fetal circulation during the last days of the pregnancy. When injections were started earlier in gestation, most of the transfer still took place after E13 (data not shown). Three offspring out of a total of 27 from two dams injected for eight days with MG plasma showed limb and cranial deformities (see later here), but the remaining fetuses appeared normal.
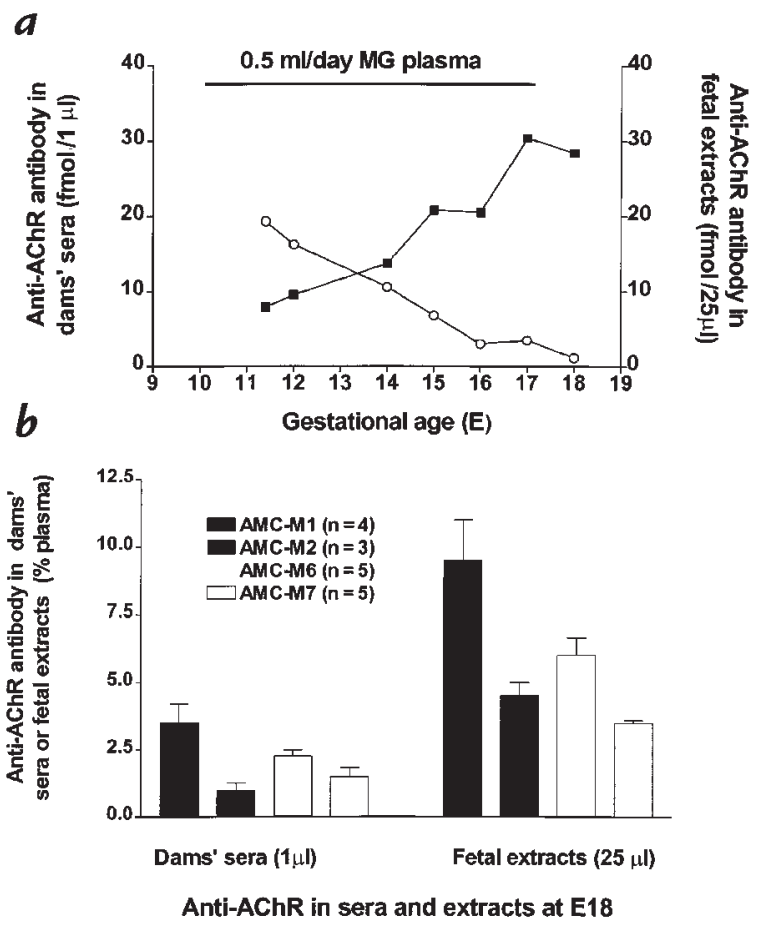

Figure 2

Transfer of human anti-acetylcholine receptor (anti-AChR) antibodies from dam to fetus. (a) Pregnant dams were injected daily with $0.5 \mathrm{ml}$ of plasma from a typical, nongravid, patient with myasthenia gravis $(M G)$, containing $90 \mathrm{nmol} / \mathrm{l}$ of anti-AChR. At different time points, anti-AChR antibody was measured in $1 \mu \mathrm{l}$ of the dams' sera and $25 \mu \mathrm{l}$ of extracts of their fetuses. Subsequent analysis showed that $25 \mu \mathrm{l}$ of fetal extracts (from $12.5 \mu$ l of fetal tissue) was equivalent to $3.5 \mu \mathrm{l}$ of fetal serum. Thus, at E18, the fetal serum would have contained about $8 \mathrm{fmol} / \mu \mathrm{l}$ of antiAChR. Similar results were obtained in two other experiments, and raised anti-AChR levels were also detected in the fetal yolk sacs. (b) Anti-AChR antibodies measured in sera and fetal extracts taken at E18/19 from dams injected from E9 to E16 with AMC-M plasmas. 


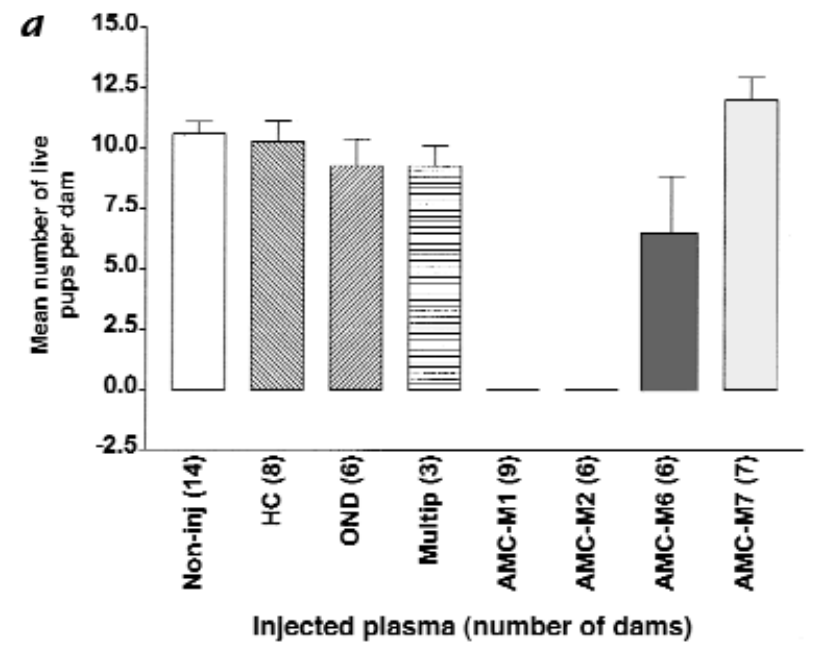

$\boldsymbol{b}$

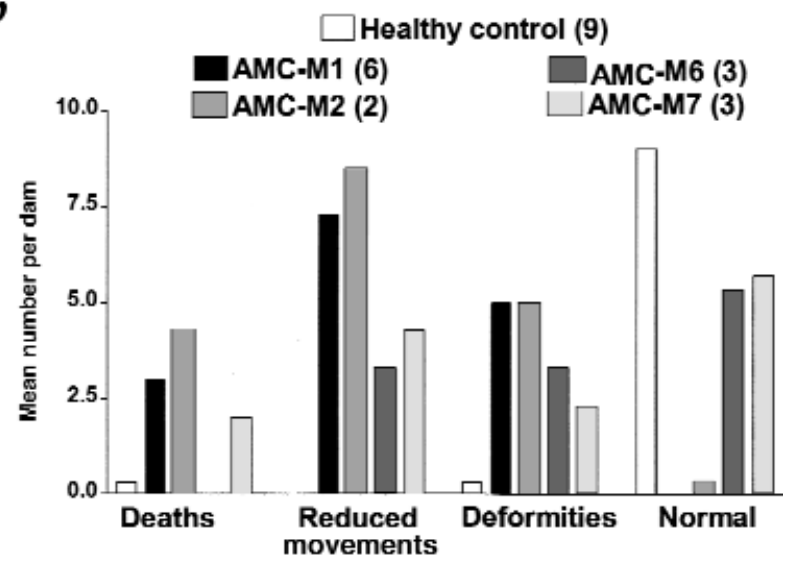

Figure 3

Results of injecting plasmas into pregnant dams. (a) Mice allowed to give birth spontaneously. The mean number ( \pm SEM) of live pups born to each uninjected dam, or dams injected with plasmas from controls (HC, healthy; OND, other neurologic diseases; two dams each with plasma from patients with Guillain-Barré syndrome, Miller Fisher syndrome, and acquired neuromyotonia; Multip, healthy individuals with two or more children) are compared with those injected with plasmas from AMC mothers. (b) In utero and immediate ex utero observations. The mean number of fetuses that had undergone intrauterine death, showed reduced movements or obvious deformities, or appeared normal is shown. Number of dams investigated are given in brackets. $\ln b$, there was marked variability between different dams, and SEMs are not given.

We then injected the pregnant dams with AMC-M plasma preparations and measured the anti-AChR levels in the dams and the fetuses at E18, just before spontaneous delivery. In these preliminary studies, it was evident that many of the fetuses were affected by the antibody transfer (see next). At this stage of the pregnancy, the levels of antibody in the dams were between $1 \%$ and $3 \%$ of that in the injected plasmas, and the fetal extracts contained equivalent amounts (Figure $2 b$ ). Again, the dams did not show any adverse effects.

Effects of transfer of AMC-M plasma IgG. To test the effects of transfer of AMC-M antibodies on fetal development, we injected $0.5 \mathrm{ml}$ of AMC-M and control plasmas daily into pregnant dams from E9 to E16 and com- pared the number of live pups with that from uninjected controls. Plasma from healthy individuals, from other patients with immune-mediated neurologic disorders (Guillain-Barré syndrome, Miller Fisher syndrome, acquired neuromyotonia), or from three healthy multiparous women did not affect the average number of live pups delivered (Figure $3 a$ ). By contrast, no live pups were born to nine AMC-M1- or six AMCM2-injected dams; in many cases, spontaneous delivery was delayed until E20 or E21, when dead pups were born or removed under anesthesia. Plasma from AMCM6 had a much more variable effect, but overall, the number of live pups, 39 from six dams, was less than that from control-injected dams. Plasma from AMC-M7 had no apparent effect. The mean results for each plasma are shown in Figure $3 a$. One litter of pups born to a dam treated with plasma from the patient with Guillain-Barré syndrome did not thrive, but otherwise, those pups that were born normal, thrived, gained weight, and showed no ill effects over the following six weeks.

In further studies, we sacrificed the dams at E18 or E19 and examined the fetuses in utero and then ex utero. All AMC-M-injected dams had some intrauterine deaths, varying in apparent gestational age at death (based on size) from about E13 to E16, but no such deaths were present in control-injected dams (Figure $3 b$ ). The control-treated fetuses were healthy and showed spontaneous movements in utero. By contrast, many of those from AMC-M-treated dams moved very little or only in response to mechanical stimulation. On removal from the uterus, many were clearly deformed, with the average number of affected pups varying from 2.3 to 5.0 per pregnancy (Figure $3 b$ ) and showing no movements or only occasional respiratory efforts.

The fetuses were then examined in detail and photographed before or after fixation. Untreated and control plasma-treated pups adopted a posture ex utero as illustrated in Figure $4 a$ (left). Many AMC-M-treated pups demonstrated fixed lower or upper limbs and crooked, misplaced tails (Figure $4 a$, right; $b$ and $c$ ). Abnormalities of the spine were frequent and included kyphoscoliosis and torticollis (Figure 4, b and $c$ ). In some cases, there was evidence of edema (Figure $4 d$, right) and loss of normal skin creases (Figure 4e, right). Many fetuses had more than one abnormality. Table 2 summarizes the frequency of the main features.

To demonstrate that the effects were due to transfer of IgG antibodies, we first injected purified IgG from AMCM1 and AMC-M2 into pregnant dams, starting at E13 to conserve material. The number of surviving offspring was significantly reduced in both cases (AMC-M1: $4.7 \pm$ 1.2 (3), $P=0.02$; AMC-M2: $4.0 \pm 2.0$ (3), $P=0.023)$, compared with noninjected controls $(9.7 \pm 1.0(6)$; mean \pm SEM $(n)$, unpaired $t$ test), even though only four to five injections had been given. However, because of fears that elution from the protein $\mathrm{G}$ column might have partially damaged the Fc regions and reduced transfer, we subsequently used crude ammonium sulfate precipitates of IgG from AMC-Ms and a healthy control to study the effects of transfer. The transfer of IgG was efficient, and many of the fetuses observed at E18/19 were abnormal (Table 2). Control IgG-treated pups were born spontaneously and were normal. 
Table 2

Numbers of fetuses with different abnormalities taken ex utero from dams treated with AMC-M plasma

\begin{tabular}{|c|c|c|c|c|c|c|}
\hline $\begin{array}{l}\text { Plasma, } \\
\text { no. dams } \\
\text { (no. fetuses) }\end{array}$ & $\begin{array}{l}\text { Reduced } \\
\text { movements }\end{array}$ & $\begin{array}{l}\text { Joint } \\
\text { contractures: } \\
\text { upper limbs }\end{array}$ & $\begin{array}{c}\text { Joint } \\
\text { contractures: } \\
\text { lower limbs }\end{array}$ & $\begin{array}{l}\text { Spinal deformities } \\
\text { kyphoscoliosis } \\
\text { hyperextension of the } \\
\text { neck, torticollis }\end{array}$ & $\begin{array}{l}\text { Cranio-facial } \\
\text { abnormalities }^{B}\end{array}$ & $\begin{array}{l}\text { Skin abnormalities } \\
\text { oedema, decreased } \\
\text { skin creases, } \\
\text { webbing }\end{array}$ \\
\hline AMC-M1, 4 (29) & 29 & 17 & 11 & 13 & 5 & 12 \\
\hline $\operatorname{lgG}, 1(10)$ & 9 & 3 & 5 & 6 & 0 & 0 \\
\hline AMC-M2, 2 (17) & 17 & 9 & 8 & 2 & 1 & 6 \\
\hline $\operatorname{lgG}, 1$ (12) & 12 & 4 & 5 & 8 & 1 & 7 \\
\hline AMC-M6, 3 (26) & 10 & 11 & 9 & 4 & 0 & 3 \\
\hline $\operatorname{lgG}, 1(11)$ & 11 & 4 & 3 & 8 & 0 & 0 \\
\hline AMC-M7, 3 (30) & 13 & 5 & 6 & 2 & 1 & 3 \\
\hline $\lg G, 1(13)$ & 1 & 0 & 1 & 0 & 0 & 0 \\
\hline \multicolumn{6}{|l|}{$\operatorname{lgG}, 3(24)^{\mathrm{D}}$} & 0 \\
\hline \multicolumn{7}{|c|}{ Alncludes complete paralysis and lack of spontaneous movements; some fetuses responded to mechanical stimulation. } \\
\hline \multicolumn{7}{|c|}{ BIncludes pointed facies, or low-set ears. } \\
\hline \multicolumn{7}{|c|}{$\begin{array}{l}\text { ¿Controls include } 72 \text { fetuses of control-injected dams and } 38 \text { fetuses of noninjected dams. Five of the } 72 \text { fetuses of control-injected dams had mild contractures } \\
\text { in a single distal joint. }\end{array}$} \\
\hline
\end{tabular}

Histological features in affected fetuses. Several of the fetuses had craniofacial abnormalities such as long or pointed facies and low-set ears, and three affected fetuses were examined after paraffin embedding. Histological examination of a fetus injected with plasma from the nongravid MG patient showed compression and reduced size of the brain associated with an increased amount of fluid around it (Figure $5 b$ ). An Arnold-Chiari-like malformation with herniation of the medulla oblongata (Figure $5 d$ ) was found in an AMC-M6-treated fetus, and an atrial septal defect was noted in a fetus from a dam treated with AMC-M7 plasma (Figure 5f). These changes were not seen in healthy control plasma-treated or untreated animals (Figure 5, $a, c$, and $e$ ).

The results in Table 2 show that many of the fetuses of dams treated with AMC-M preparations were affected, but the lethality and severity of the conditions did not appear to correlate either with the antibody titers against human fetal AChR in the maternal serum (Table 1) or with titers against mouse fetal AChR (data not shown).

Pathogenic antibodies in an anti-AChR antibody-negative mother with AMC. During the course of this study, we were sent serum from a mother who had four consecutive pregnancies with fatal AMC, consistently with an antibody-mediated pathogenesis, but who did not have detectable anti-AChR. Injection of $0.5 \mathrm{ml} /$ day of her serum from E9 to E16 produced three intrauterine deaths and four affected fetuses from a total number of 23 implantations (Figure 6, $a$ and $b$ ), indicating the involvement of a transferable serum factor.

\section{Discussion}

To explore a possible pathogenic role of human maternal antibodies on fetal development, we have developed an animal model in which human antibodies are transferred from the maternal to the fetal circulation. We started with samples from four women who were known to have high levels of antibodies to fetal AChR and who had given birth to two or more babies with severe, usually fatal, AMC. We found that the transfer of human antibodies to the mouse fetus is efficient, leading to substantially raised titers in the fetuses. Transfer of plasma antibodies from the mothers with AMC produced fixed joints and other deformities in the mouse fetuses that were similar to those observed in human babies with AMC, and plasmas and purified IgG caused substantial fetal death. We also found evidence of a transferable serum factor from one other AMC mother who did not have detectable anti-AChR antibodies. Thus, although our results confirm that maternal antibodies, probably those directed at fetal AChR (see later here), are a cause of AMC in some cases, they suggest that this condition may sometimes be caused by other antibodies or by other serum factors.

AMC is a condition characterized by contractures in more than one joint, but it is frequently associated with other, sometimes life-threatening, developmental abnormalities frequently including pulmonary hypoplasia (when it is referred to as Pena-Shokeir syndrome; ref. 10). AMC can be caused by genetic factors, infectious agents, drugs and toxins, physical agents, and maternal illness, particularly MG and multiple sclerosis $(1,2)$. Hall (1) divided AMC cases into three groups: (a) those with predominantly limb involvement who tend to survive; the condition is often nonprogressive (suggesting an environmental etiology), although even with extensive surgery and physiotherapy the patients may be disabled for life; (b) those with additional involvement of other systems; and (c) those in which there is also CNS involvement with mental retardation in the survivors. Cases with associated maternal illness, such as MG, were considered a separate group.

The causes of AMC are many, but the common final pathway appears to be lack or restriction of fetal movement in utero (5-7). Paralysis of the chick embryo with curare leads to joint contractures (5), and injection of curare into rats embryos at E17-20 (last three days of 
$a$

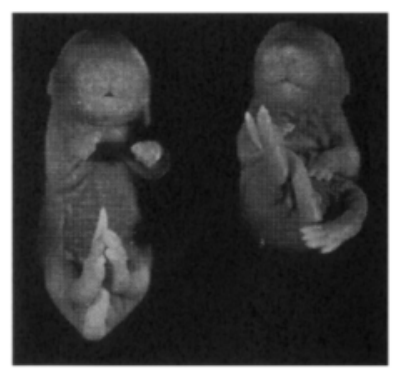

$\boldsymbol{b}$

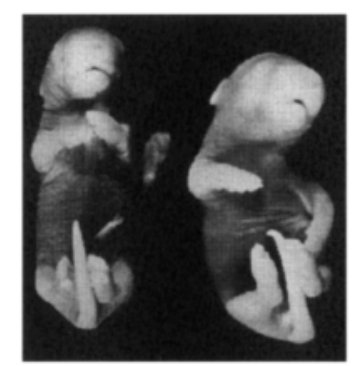

$c$

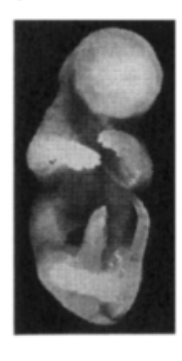

$d$

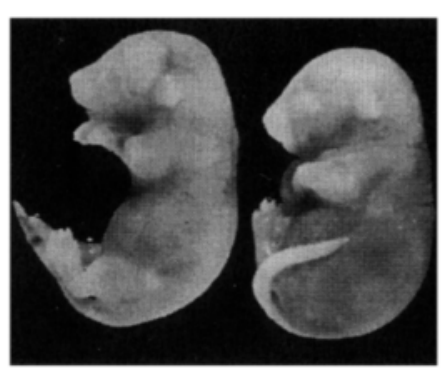

$\boldsymbol{e}$

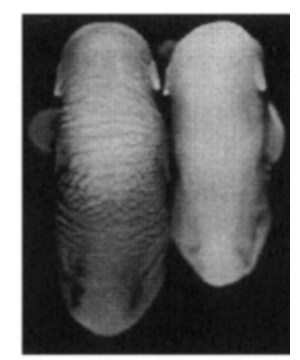

Figure 4

Fixed deformities found in fetuses taken at E18 or E19 from plasma-injected mouse dams. (a) A control plasma-treated (left) and AMC-M1-treated fetus (right) removed at E19 and fixed in 95\% ethanol. The AMC-M-treated fetus shows severe external rotation of the hip, genu varum, adduction and varus of the forefoot, with adduction of the right forelimb, ankylosis of the elbow, and varus of the left forelimb. ( $\boldsymbol{b}$ ) Control (left) and AMCM7-treated (right) fetuses removed at E18 and fixed in 95\% ethanol. There is severe torticollis, asymmetry of the shoulders, internal rotation and abduction of the left forelimb, and scoliosis of the spine. The lower limbs are normal. (c) A fetus from an AMC-M1-injected dam, removed at E18 and not fixed before photography. There is internal rotation of the left hip associated with genu varum and internal rotation of the foot; the tail is misplaced in relation to the hindlimbs. The head is flexed both laterally and frontally. (d) Fetuses from control (left) and AMC-M-treated (right) dams at E18. The latter shows marked hydrops, the left wrist is fixed in flexion, and the tail is displaced. (e) Fetus from AMC-M1-treated dam (right) has cutaneous edema with absence of normal skin creases that are evident in the control (left).

gestation in the rat) produced contractures, lung hypoplasia, micrognathia, and polyhydramnios, similar to the changes reported here (6), which were termed the fetal akinesia deformation sequence. Moreover, arthrogryposis was reported in the baby of a woman treated during pregnancy with muscle relaxants (7). Although arthrogryposis is recognized as a rare complication of maternal MG $(13,23,24)$, the role of maternal circulating factors in causing AMC and other developmental abnormalities has not been widely considered.

MG is caused by antibodies to the AChR at the neuromuscular junction $(11,23)$. The fact the MG was not present, or was only subsequently diagnosed in three of the mothers described here, suggests that the fine specificity of the antibodies must be different from those in classical MG. Indeed, the antibodies even at high dilution strongly inhibited the function of human fetal AChR while having little effect on adult $\operatorname{AChR}(8,9)$. High titers of these fetal-specific inhibitory antibodies are rare in $\mathrm{MG}$ (9), explaining why AMC is uncommon in babies born to typical MG mothers. AMC-M plasmas also inhibited mouse fetal AChR function (Vincent, A., unpublished data), and the inhibitory activity was highest in AMC-M1 and AMC-M2 and least in AMC-M6 and AMC-M7, correlating with the severity of the effects that we observed in the fetuses. Thus, it is very likely that the lack of fetal movement in the mouse fetuses, and the subsequent deformities, were due to antibodies inhibiting mouse fetal AChR.

AMC-M1, AMC-M2, and AMC-M3 had not previously been diagnosed as having MG, suggesting that antiAChR antibodies should be looked for in any unexplained case of AMC. Moreover, the absence of detectable antibodies to AChR should not exclude the possibility of a transferable factor; our finding of limb and spinal fixed deformities in a few of the fetuses transferred with serum from a healthy woman who was antiAChR antibody-negative suggests that there are other antibodies (or other serum factors) that can be responsible for fetal abnormalities. Indeed, any neonatal or fetal condition that shows a high recurrence rate without clear evidence of a genetic basis would be a candidate for testing in this experimental model, and demonstration of an appropriate effect on the fetus would stimulate the search for a specific autoantibody or other factor.

Passive transfer to mice has proved to be an essential tool in demonstrating a pathogenic role of serum antibodies in peripheral neurologic disease, particularly MG $(11,21,22)$ and other disorders caused by autoantibodies to peripheral ion channels (23), but MG plasmas seldom produce overt clinical disease when injected into adult mice (22). The plasma from a 15-year-old, nongravid patient with MG, whom we studied for anti-AChR transfer (Figure 2a), produced a small number of affected fetuses, one of which demonstrated reduced brain size (Figure $5 b$ ). It could be that transfer of human antibodies to the developing mouse (or possibly neonatal transfer via the milk, which is very effective; Jacobson, L., and Vincent, A., unpublished results), because of greater accessibility or vulnerability of the nervous system, will prove to be a more sensitive approach to detect pathogenic antibodies in some adult neurologic conditions.

Transfer of IgG across the placenta is known to be an active process, dependent on specific Fc receptors (25), and it was not a priori expected that the transfer of human antibodies to the mouse fetus would be efficient; in fact, the transfer probably occurs via the yolk sac rather than the placenta (26), and we found raised levels of anti-AChR in the fetal yolk sac fluid (data not shown). There have been very few previous studies on transfer of human antibodies to the mouse fetus, although injection of anti-cardiolipin antibodies into pregnant mice resulted in reduced numbers of pregnancies and reduced fetal size $(27,28)$, and in another study, some transfer of human antinuclear antibodies from dam to fetus was demonstrated (29).

There are several other conditions in which maternal antibodies are known to be pathogenic, including hemolytic disease of the newborn, due to the transfer of antibodies against the rhesus antigen on fetal red 

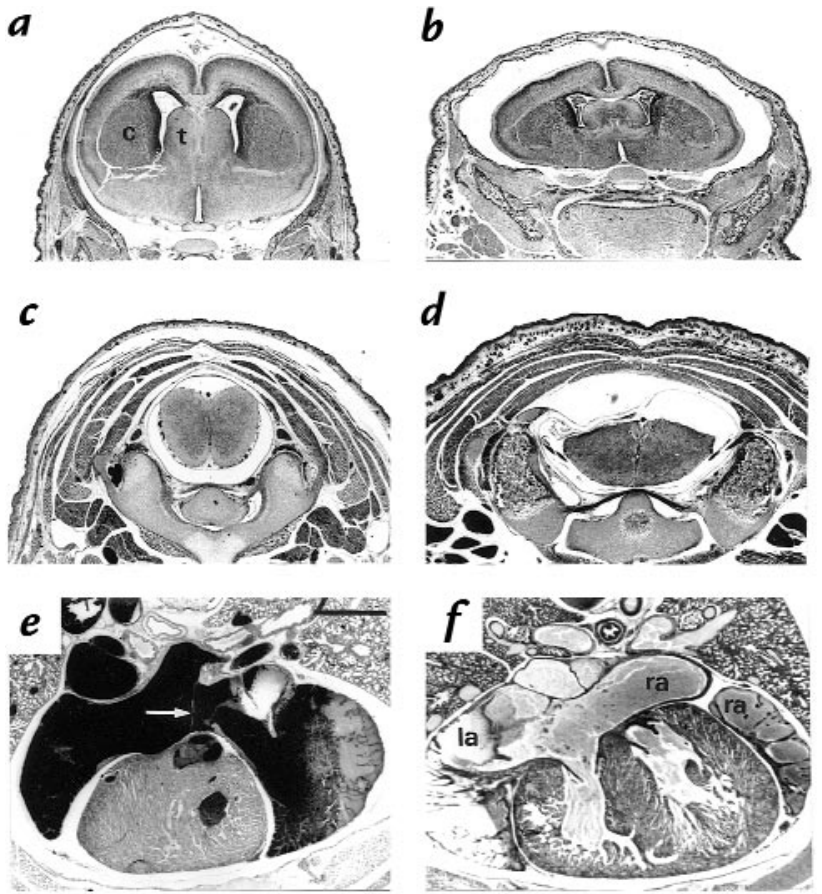

Figure 5

Internal abnormalities in mouse fetuses $(b, d$, and $f$ ) compared with healthy plasma-injected ( $a$ and $c$ ) or noninjected $(e)$ fetuses. ( $\boldsymbol{a}$ and $\boldsymbol{b}$ ) Coronal sections of the forebrain at the level of the striatum, caudate nucleus, and thalamus; all the normal structural features are present in the affected fetus (nongravid MG plasma-injected; $b$ ), but the brain is dorsoventrally compressed and reduced in size. ( $\boldsymbol{c}$ and $\boldsymbol{d}$ ) Transverse sections of the neck at the atlas/axis level; the affected fetus (AMCM6-injected; $d$ ) shows an Arnold-Chiari-like malformation with the medulla oblongata herniated into the spinal canal. ( $\boldsymbol{e}$ and $\boldsymbol{f}$ ) Transverse sections of the heart. The affected fetus (AMC-M7-injected; $f$ ) shows an atrial septal defect. $c$, head of caudate nucleus; la, left atrium; ra, right atrium; $t$, thalamus. Scale bar: $1 \mu \mathrm{m}$. $a$

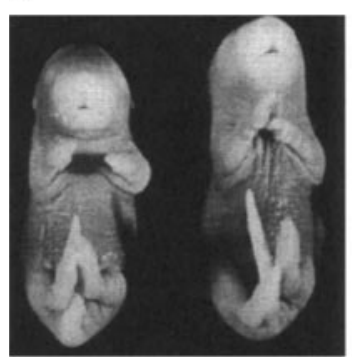

$b$

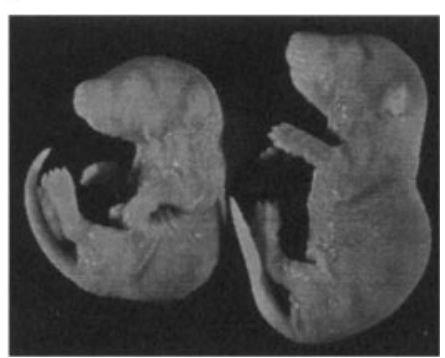

Figure 6

Deformities in fetuses treated with serum from a mother with AMC without detectable anti-AChR antibodies. (a) Fetuses from healthy control-injected (left) and AMC-M8-treated (right) dams. The AMCM8-treated fetus has a mild extension of the right hip, and hyperextension of the head with adduction of the forelimbs. (b) Two AMC-M8-treated fetuses, fixed in Bouin's solution, showing pointed facies, lowering and adduction of the shoulder and flexion of the hip joints (left), and hyperextension of the cervical spine with lumbar kyphosis (right). blood cells $(30,31)$; neonatal idiopathic thrombocytopenia purpura (32); and heart block that results from transfer of antibodies against Ro or La antigens $(33,34)$. Interestingly, in the congenital heart block, the mother may have systemic lupus erythematosus but is often asymptomatic at the time. This suggests that the immune response may be specifically directed at fetal antigens but that subsequent maternal disease, if it occurs, is due to the spreading of the immune response to include maternal autoantigens; this may also apply in the cases described here. Our model should be useful for further investigations of these conditions.

Antibody-mediated disorders can be treated by a variety of immunotherapies, including plasma exchange, intravenous Ig, and corticosteroids, and these treatments can be used during pregnancy. Both AMC-M2 and AMC-M6 have produced healthy babies after such treatments, as reported previously in a similar case (35). Women whose fetuses are at risk from hemolytic anemia (31) or neonatal idiopathic thrombocytopenic purpura (32) have also been treated successfully with intravenous IgG. This treatment may be particularly appropriate because it specifically competes with the maternal IgG for transfer across the placenta (36). Identification of an immune basis for any developmental disorder should be an indication for immunotherapy during subsequent pregnancies.

It has been proposed previously that maternal antibodies might be a cause of neurodevelopmental disorders $(16,17)$. Mice immunized against brain emulsions or phospholipid antigens were examined for embryo survival and morphology (16), and a possible role of maternal antibodies in dyslexia and other disorders was proposed (17). In the New Zealand black mouse, the uterine environment is responsible for some behavioral abnormalities (whereas others are due to genetic factors), and it seems likely that autoantibodies are involved (37); moreover, a recent study shows that transfer of mouse monoclonal antiphospholipid antibodies to the mouse fetus causes behavioral differences (38), clearly suggesting that antibodies transferred during development can affect the CNS as well as the periphery. The effects on brain structure that we found in a limited study of affected fetuses suggest that transfer of human maternal antibodies can also cause, either directly or indirectly, pathology to the CNS.

In conclusion, our results show that human antibodies can be transferred from the mouse dam to her fetuses, and confirm that arthrogryposis can be caused by maternal antibodies, particularly those directed at the fetal AChR. The experimental model that we have established can be used to investigate an antibody-mediated pathology in other cases of AMC and, more importantly, in other congenital disorders. This approach could have wide application to the study of fetal or neonatal development and the disorders that affect it. It may also provide a sensitive test for pathogenic antibodies against neuronal antigens in adult neurologic disorders and will provide a useful model in which to test possible therapeutic approaches to limit transfer of pathogenic antibodies. 


\section{Acknowledgments}

We are grateful to John Newsom-Davis, Louise Brueton, and Susan Huson for clinical data and plasma samples. This study was supported by grants from the Myasthenia Gravis Association/Muscular Dystrophy Group (to Louise Jacobson) and the Medical Research Council of Great Britain (to Agata Polizzi and Angela Vincent). Agata Polizzi was also funded by a European Community grant from the Division of Paediatric Neurology/Human Embryology of the University of Catania.

1. Hall, J.G. 1996. Arthrogryposes: multiple congenital contractures. In Principles of medical genetics. D.L. Rimoin, J.M. Connor, and R.E. Pyeritz, editors. Churchill Livingstone. New York, NY. 2869-2915.

2. Porter, H.J. 1995. Lethal arthrogryposis multiplex congenita (fetal akinesia deformation sequence, FADS). Pediatr. Pathol. Lab. Med. 15:617-637.

3. Dastur, D., Rassak, Z., and Bharucha, E. 1972. Arthrogryposis multiplex congenita. Part 2: Muscle pathology and pathogenesis. J. Neurol. Neurosurg. Psychiatry. 35:435-450.

4. Banker, B. 1985. Neuropathologic aspects of arthrogryposis multiplex congenita. Clin. Orthop. 194:30-43.

5. Drachman, D.B. and Coulombre, A. 1962. Experimental clubfoot and arthrogryposis multiplex congenita. Lancet. 2:523-526.

6. Moessinger, A. 1983. Fetal akinesia deformation sequence: an animal model. Pediatrics. 72:857-863.

7. Jago, R.H. 1970. Arthrogryposis following treatment of maternal tetanus with muscle relaxants. Arch. Dis. Child. 45:277-279.

8. Vincent, A., et al. 1995. Arthrogryposis multiplex congenita with maternal autoantibodies specific for a fetal antigen. Lancet. 346:24-25.

9. Riemersma, S., et al. 1997. Association of arthrogryposis multiplex congenita with maternal antibodies inhibiting fetal acetylcholine receptor function. J. Clin. Invest. 98:2358-2363.

10. Pena, S.D.J., and Shokeir, M.H.K. 1974. Syndrome of camptodactyly, multiple ankyloses, facial anomalies and pulmonary hypoplasia: a lethal condition. J. Pediatr. 85:373.

11. Drachman, D.B. 1994. Myasthenia gravis. New Engl. J. Med. 330:1797-1810

12. Venet-der Garabedian, B., et al. 1994. Association of neonatal myasthenia gravis with antibodies against the foetal acetylcholine receptor. $J$. Clin. Invest. 94:555-559.

13. Eymard, B. 1997. Neonatal myasthenia gravis clinical and pathophysiological aspects. Adv. Organ Biol. 2:235-247.

14. Barnes, P.R.J., et al. 1995. Recurrent congenital arthrogryposis leading to a diagnosis of myasthenia gravis in an initially asymptomatic mother. Neuromuscul. Disord. 5:55-65.

15. Hesselmans, L., Jennekens, F., van den Oord, C., Veldman, H., and Vincent, A. 1993. Immunoreactivity to the acetylcholine receptor in developing human muscle. Anat. Rec. 236:553-562.

16. Gluecksohn-Waelsch, S. 1957. The effect of maternal immunization against organ tissues on embryonic differentiation in the mouse. $J$. Embryol. Exp. Morphol. 5:83-92.

17. Adinolfi, M. 1993. Fetal exposure to maternal brain antibodies and neurological handicap. In Dyslexia and development. A.M. Galaburda, editor. Harvard University Press. Cambridge, MA. 155-167.

18. Vincent, A., Jacobson, L., and A. Polizzi. 1997. An animal model for arthrogyrposis multiplex congenita caused by antibodies to fetal acetylcholine receptor. Ann. Neurol. 42:391A. (Abstr.)

19. Jacobson, L., Beeson, A., and Vincent, A. 1998. An animal model of maternal antibody-mediated arthrogryposis multiplex congenita. Ann. NY Acad. Sci. 841:565-567.

20. Beeson, D., Jacobson, L., Newsom-Davis, J., and Vincent, A. 1996. A transfected human muscle cell line expressing the adult subtype of the human muscle acetylcholine receptor for diagnostic assays in myasthenia gravis. Neurology. 47:1552-1555.

21. Toyka, K.V, et al. 1977. Myasthenia gravis: study of humoral immune mechanisms by passive transfer to mice. New Engl. J. Med. 296:125-131.

22. Mossman, S., Vincent, A. and Newsom-Davis, J. 1988. Passive transfer of myasthenia gravis by immunoglobulins: lack of correlation between antibody bound, acetylcholine receptor loss and transmission defect. $J$. Neurol. Sci. 84:15-28.

23. Vincent, A. 1997. Disorders of the human neuromuscular junction. Adv. Organ Biol. 2:315-349.

24. Dinger, J., and Prager, B. 1993. Arthrogryposis multiplex in a newborn of a myasthenic mother - case report and literature. Neuromuscul. Disord. 3:335-339.

25. Simister, N.E. and Story, C.M. 1997. Human placental Fc receptors and the transmission of antibodies from mother to fetus. J. Reprod. Immunol. 37:1-23.

26. Ghetie, V., and Ward, E.S. 1997. FcRn: the MHC class I-related receptor that is more than an IgG transporter. Immunol. Today. 18:592-598.

27. Blank, M, Cohen, J., Toder, V., and Shoenfeld, Y. 1991. Induction of antiphospholipid syndrome in naïve mice with mouse lupus monoclonal and human polyclonal anti-cardiolipin antibodies. Proc. Natl. Acad. Sci. USA. 88:3069-3073.

28. Shoenfeld, Y., Sherer, Y., and Blank, M. 1998. Antiphospholipid antibodies in pregnancy. Scand. J. Rheumatol. Suppl. 107:33-36.

29. Guzman-Enriquez, L., Avalos-Diaz, E., and Herrera-Esparza, R. 1990. Transplacental transfer of human antinuclear antibodies in mice by injection of Lupus IgG in regnant animals. J. Rheumatol. 17:52-56.

30. Gottvall, T., Hilden, J.O., Nelson, N., and Filbey, D. 1995. Severe RhD immunization: anti-D quantitation and treatment possibilities during pregnancy and after birth. Acta. Paediatr. 84:1315-1317.

31. Deka, D., Buckshee, K., and Kinra, G. 1996. Intravenous immunoglobulin as primary therapy or adjuvant therapy to intrauterine fetal blood transfusion; a new approach in the management of severe Rh immunisation. J. Obstet. Gynaecol. Res. 22:561-567.

32. Porcelign, L., and Kanhai, H.H. 1988. Fetal thrombocytopenia. Curr. Opin. Obstet. Gynecol. 10:117-122.

33. Chameides, L., et al. 1977. Association of maternal system lupus erythematosus with congenital complete heart block. New Engl. J. Med. 297:1204-1207.

34. Olson, N.Y., and Lindsey, C.B. 1987. Neonatal lupus syndrome. Am. J. Dis. Child. 141:908-910.

35. Carr, S.R., Gilchrist, J.M., Abuelo, D.N. and Clark, D. 1991. Treatment of antenatal Myasthenia Gravis. Obstet. Gynecol. 78:485-489.

36. Urbaniak, S.J., Duncan, J.I., Armstrong-Fisher, S.S. Abramovich, D.R., and Page, K.R. 1997. Transfer of anti-D antibodies across the isolated perfused human placental lobule and inhibition by high dose intravenous immunoglobulin: a possible mechanism of action. Br. J. Haematol. 98:493-494.

37. Denenberg, V.H., et al. 1991. Effects of the autoimmune uterine/maternal environment upon cortical ectopias, behaviour and autoimmunity. Brain Res. 563:114-122.

38. Ziporen, L., Shoenfeld, Y., Levy, Y., and Korczyn, A.D. 1997. Neurological dysfunction and hyperactive behaviour associated with antiphospholipid antibodies. A mouse model. J. Clin. Invest. 100:613-619. 